\title{
Stressfrakturen der unteren Extremität
}

\section{Stress Fractures of the Lower Limb}

\section{Übersicht}

Als Stressfrakturen bezeichnet man partielle oder vollständige Frakturen, welche durch eine lange andauernde, repetitive Überlastung eines eigentlich normalen Knochens ausgelöst werden. Sie werden aus diesem Grund auch häufig Ermüdungsbrüche genannt. Hiervon abzugrenzen sind Insuffizienzfrakturen, welche einen abnormal geschwächten Knochen, z. B. im Rahmen einer Osteoporose, betreffen (Harrast MA et al. Clin Sports Med 2010; 29: 399 -416).

Stressfrakturen wurden im Jahre 1855 als Erstes von Breithaupt beschrieben, einem preußischen Militärarzt, der diese Art der Verletzung bei Rekruten mit Fußschmerzen nach langen Märschen beobachten konnte (Liong SY et al. Br J Radiol 2012; 85: 1148 -1156). Stressfrakturen machen etwa $2 \%$ aller Sportverletzungen aus, wobei die Knochen der unteren Extremität in ca. $95 \%$ der Fälle betroffen sind (Liong SY et al. Br J Radiol 2012; 85: 1148 - 1156). Die typischen Lokalisationen von Stressfrakturen der unteren Extremität sind in den ( $\triangleright$ Abb. 1 - 3) schematisch dargestellt.

Pathophysiologisch bedeutsam bei der Entstehung von Stressfrakturen ist die nicht mehr ausreichende Anpassung des gesunden Knochens an eine vermehrte Belastung. Bei einem gesunden Individuum liegt ein Gleichgewicht zwischen osteoblastischen und osteoklastischen Prozessen vor, sodass sich der Knochen fortwährend den Belastungsänderungen anpassen und entsprechend umbauen kann. Kommt es über einen längeren Zeitraum zu einer repetitiven Überlastung, kann dieses Gleichgewicht gestört sein. Dies führt dazu, dass Mikrotraumen nicht mehr ausheilen können und sich Überlastungsreaktionen zunächst im Sinne eines Knochenmarködems ausbilden. Schreiten die osteoklastischen Prozesse bzw. die Schädigungen des Knochens weiter voran, entwickeln sich aus den Überlastungsreaktionen dann sog. Stressfrakturen, welche nicht nur den spongiösen, sondern auch den kortikalen Knochen betreffen können
(Harrast MA et al. Clin Sports Med 2010; 29: $399-416)$.

Klinisch manifestieren sich Stressfrakturen zunächst mit lokalisiertem Schmerz, wohingegen Schwellungen, Hämatome oder eine Überwärmung meist erst im späteren Verlauf auftreten (Pegrum J et al. BMJ 2012; 344: e2511; Dixon S et al. CurrProblDiagnRadiol 2011; 40: 29 -44).

Röntgenbilder, Skelettszintigrafie, MRT und CT sind die klassischen bildgebenden Verfahren, welche zur Diagnosestellung verwendet werden. Allerdings lassen sich periostale Reaktionen und eine angrenzende Mehrsklerosierung im Röntgenbild erst nach 3-4 Wochen nachweisen, im späteren Verlauf wird eine zur Kortikalis senkrecht verlaufende Frakturlinie dann als diagnostisch angesehen (Pegrum J et al. BMJ 2012; 344: e2511; Liong SY et al. Br J Radiol 2012; 85: 1148 -1156).

Die Skelettszintigrafie zeigt Stressfrakturen als Bereiche eines erhöhten Knochenumbaus an. Sie ist dabei hochsensitiv eine Stressfraktur innerhalb von 6-72 Stunden nach Symptombeginn nachzuweisen, allerdings ist die Spezifität sehr gering, sodass Stressfrakturen rein bildmorphologisch nicht von anderen Läsionen mit erhöhtem Knochenumbau abgegrenzt werden können. Darüber hinaus ist die Strahlenbelastung nicht unerheblich, was insbesondere für die jungen, sportlich aktiven Patienten mit der Verdachtsdiagnose Stressfraktur ein Problem darstellt (Pegrum J et al. BM] 2012; 344: e2511; Harrast MA et al. Clin Sports Med 2010; 29: 399 -416; Dixon S et al. CurrProblDiagnRadiol 2011; 40: 29 -44).

Die MRT ist die Modalität der Wahl, da sie sowohl eine hohe Sensitivität als auch Spezifität für Stressreaktionen aufweist. Sie kann bereits frühe Stressreaktionen im Sinne eines Knochenmarködems aufzeigen, dies sogar Wochen bevor periostale Veränderungen im Röntgenbild nachweisbar sind. Darüber hinaus können auch angrenzende Weichteile oder Gelenke beurteilt werden ohne den Patienten einer Strahlenbelastung auszusetzen (Pegrum J et al. BMJ 2012; 344: e2511; Liong SY et al. $\mathrm{Br}$ J Radiol 2012; 85: 1148 -1156;

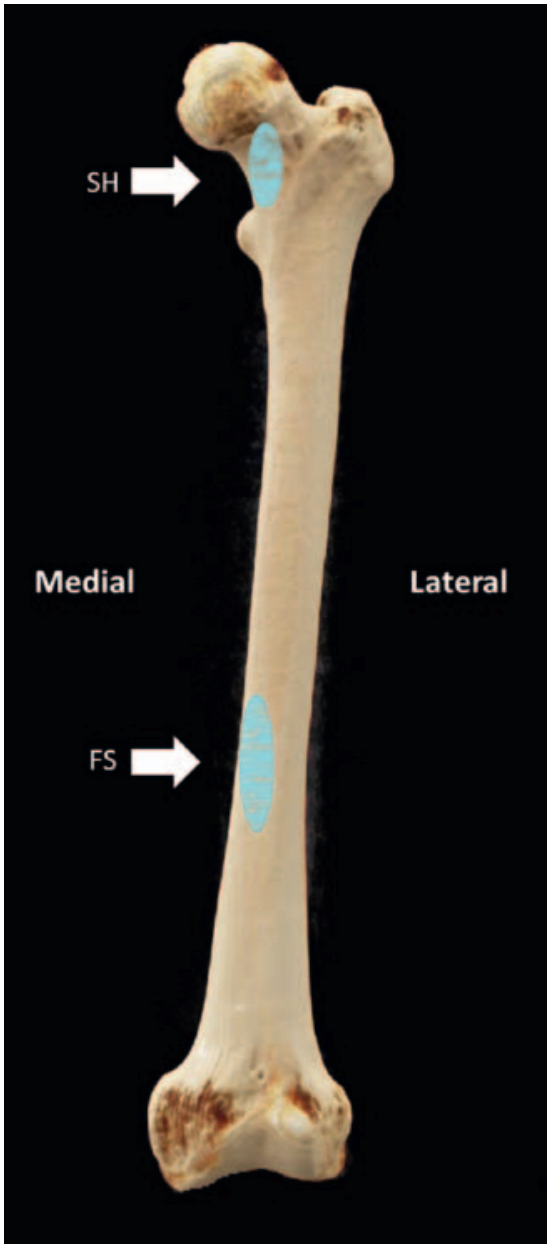

- Abb. 1 Cinematic-Rendering einer CT-Untersuchung des Femur bei einem gesunden Patienten mit den typischen Lokalisationen von Stressfrakturen. Abkürzungen: SH: Schenkelhals, FS: Femurschaft.

Dixon S et al. CurrProblDiagnRadiol 2011; 40: $29-44)$.

Die CT hat bei der Diagnose von Stressfrakturen nur eine eingeschränkte Rolle. Insbesondere die im Vergleich zur Skelettszintigrafie oder MRT geringe Sensitivität in der frühen Phase ist hier, neben der Strahlenbelastung, als Nachteil anzusehen. Im weiteren Verlauf erlaubt die CT dann jedoch die genaue Darstellung der Frakturlinie und kann ggf. zur präoperativen Planung verwendet werden. 


\section{Stressfrakturen der unteren Extremität}

\section{Schenkelhals}

Die Stressfraktur des Schenkelhalses ist insgesamt relativ selten und kam z. B. in einer Studie mit 1049 Teilnehmern in nur $5 \%$ der Fälle vor. Klinisch zeigt sich ein relativ plötzlich einsetzender Leistenschmerz, welcher sich bei Belastung verstärkt (Harrast MA et al. Clin Sports Med 2010; 29: 399 -416).

Der Schenkelhals ist hohen Belastungen bis hin zum mehrfachen des Körpergewichts ausgesetzt und hält dabei enormen Zug- und Druckbelastungen stand. Die Zugbelastungen betreffen dabei vornehmlich den cranialen-lateralen Aspekt, die Druckbelastungen den caudalenmedialen Anteil des Schenkelhalses (Harrast MA et al. Clin Sports Med 2010; 29: 399 -416; Egol KA et al. ClinOrthopRelat Res 1998; 348: 72 - 78) [ Stressfraktur des Schenkelhalses entsteht typischerweise im Bereich der Druckbelastung. Röntgenologisch zeigt sich meist nach mehr als 2 Wochen eine sklerotische Linie senkrecht zur inferioren-medialen Kortikalis. Eine frühzeitige Diagnosestellung ist mit der MRT oder Skelettszintigrafie möglich.

\section{Femurschaft}

Die Stressfraktur des Femurschafts stellt ca. 3-20\% aller Stressfrakturen bei Athleten dar (Harrast MA et al. Clin Sports Med 2010; 29: 399 -416). Sie betrifft meist den hinteren medialen Anteil des mittleren Femurschafts, zwischen der Insertion des M. adductor brevis und dem Ursprung des M. vastus medialis (Liong SY et al. $\mathrm{Br}$ J Radiol 2012; 85: 1148-1156). Klinisch manifestiert sie sich als schleichender Schmerz im Bereich von Hüfte, Oberschenkel oder Knie. Die Diagnose lässt sich, im Gegensatz zum Röntgenbild, frühzeitig mit der MRT oder Skelettszintigrafie stellen (Harrast MA et al. Clin Sports Med 2010; 29: 399 - 416) [ A Abb.5]).

\section{Tibia}

Die Stressfraktur der Tibia repräsentiert etwa $50 \%$ aller Stressfrakturen bei Athleten (Harrast MA et al. Clin Sports Med 2010; 29: 399 -416). Das Spektrum der

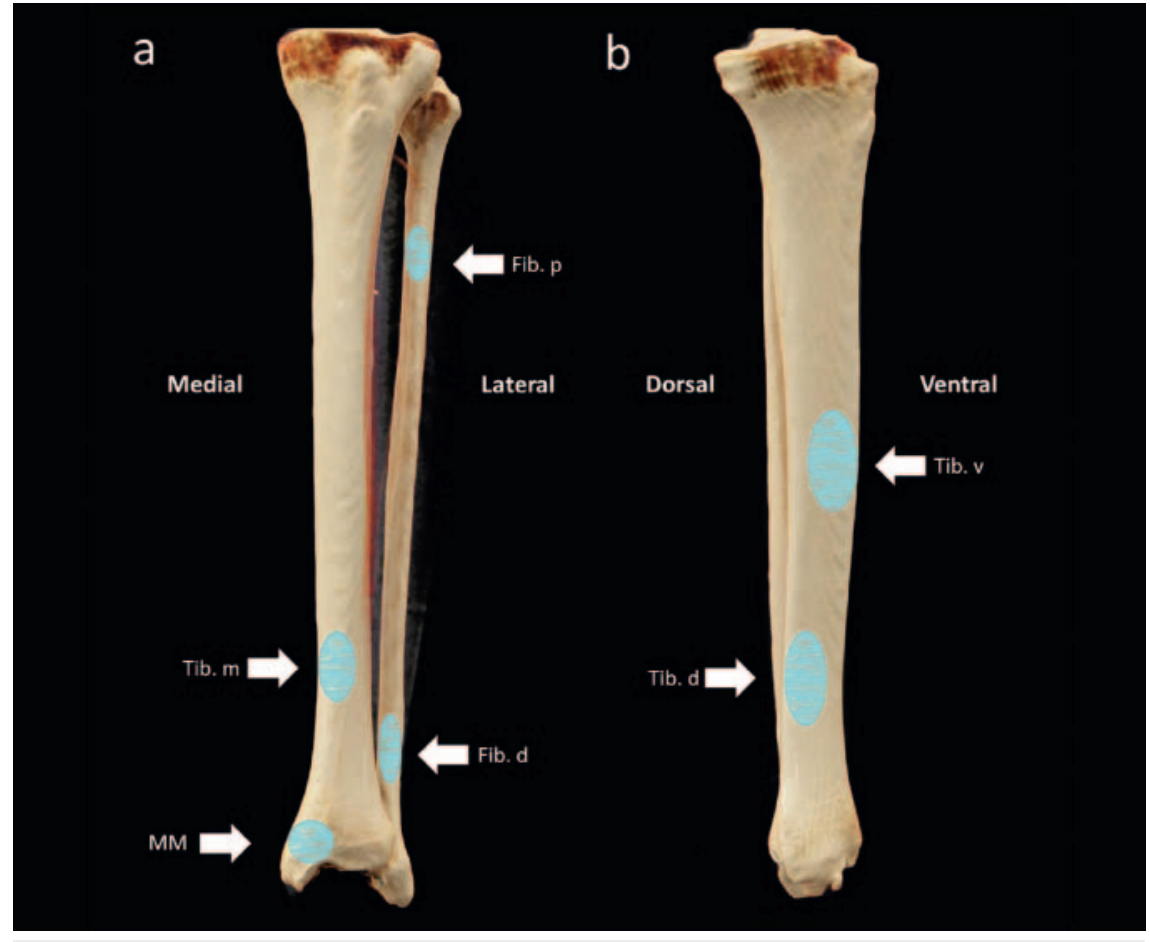

- Abb. 2 Ventrale a und mediale b Ansicht eines Cinematic-Rendering einer UnterschenkelCT-Untersuchung bei einem gesunden Patienten mit den typischen Lokalisationen von Stressfrakturen. Abkürzungen: Tib. m: Tibia medial, Fib. p: Fibula proximal. Fib, d: Fibula distal, MM: Malleolus medial, Tib. d: Tibia dorsal, Tib. v: Tibia ventral.

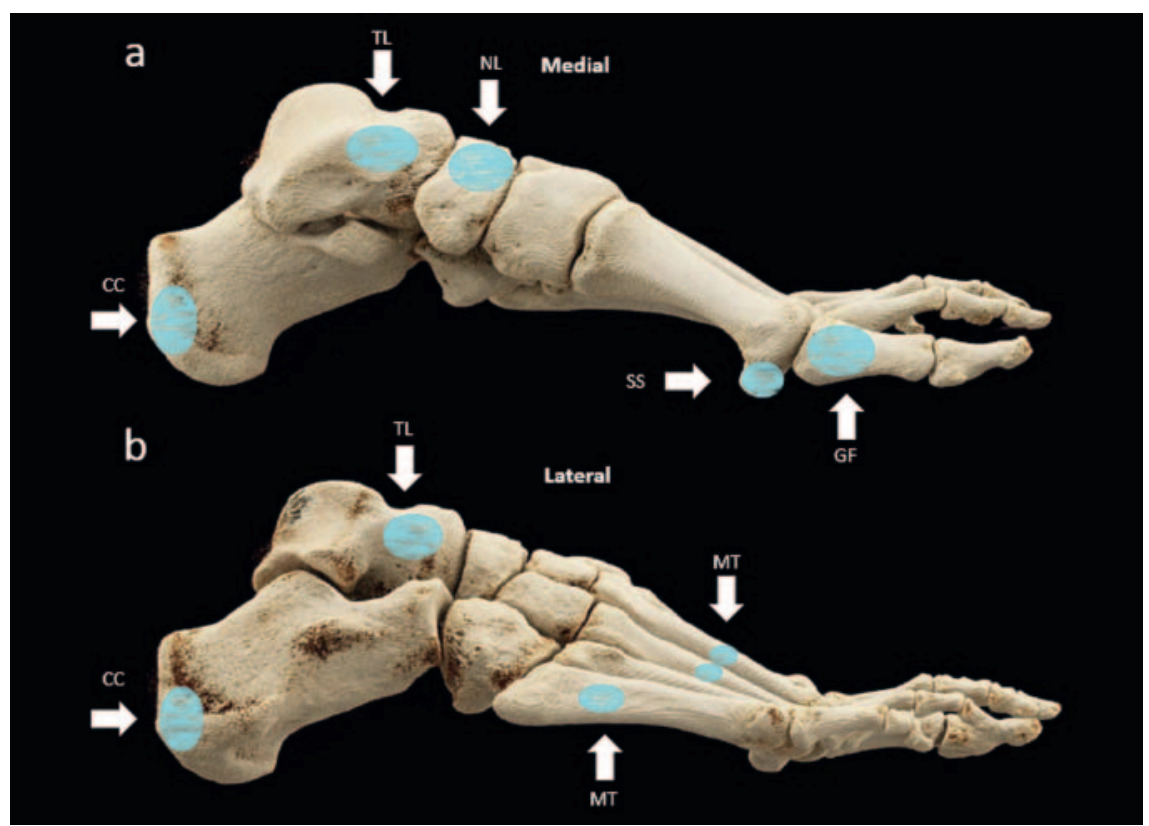

- Abb. 3 Mediale a und laterale b Ansicht eines Cinematic-Rendering einer Fuß-CT-Untersuchung bei einem gesunden Patienten mit den typischen Lokalisationen von Stressfrakturen. Abkürzungen: CC: Calcaneus, TL: Talus, NL: Os naviculare, SS: Sesambein, GF: Proximale Grundphalanx der Großzehe, MT: Metatarsalia.

Stressreaktionen reicht vom periostalen Ödem über ein ausgedehntes Knochenmarködem hin zur demarkierten Frakturli- nie (Liong SY et al. Br J Radiol 2012; 85: 1148 - 1156; Fredericson M et al. Am J Sports Med 1995; 23: $472-481)$. Die 
Mehrzahl der Fälle betrifft den posteromedialen Aspekt der distalen 2/3 des Tibiaschafts und wird klinisch als Schienbeinschmerz mit Verstärkung bei Belastung manifest (Liong SY et al. Br J Radiol 2012; 85: 1148-1156) [ A Abb. 6, 7]). Bei vermehrter Zugbelastung hingegen ist meist der anteriore Aspekt betroffen, was sich als unspezifischer Unterschenkelschmerz manifestiert (Harrast MA et al. Clin Sports Med 2010; 29: $399-416$; Liong SY et al. Br J Radiol 2012; 85: 1148-1156). In den meisten Fällen kommt es zu einer horizontalen Frakturlinie senkrecht zur Kortikalis, selten auch zu longitudinalen Fissuren, welche dann häufig nur im CT sichtbar sind (Harrast MA et al. Clin Sports Med 2010; 29: $399-$ 416; Liong SY et al. Br J Radiol 2012; 85: 1148 - 1156).

\section{Medialer Malleolus}

Die Stressfraktur des medialen Malleolus kommt nur selten vor und zeigt sich klinisch als lokalisierter Schmerz, Druckschmerzhaftigkeit und Schwellung im Bereich des Innenknöchels (Harrast MA et al. Clin Sports Med 2010; 29: 399 - 416; Menge TJ et al. J Foot Ankle Surg 2015; 54: 242 -246). Typischerweise entstehen Stressfrakturen in diesem Bereich am Übergang vom Innenknöchel zum Pilon tibiale als inkomplette Fraktur senkrecht zur Kortikalis der Gelenkfläche (Harrast MA et al. Clin Sports Med 2010; 29: 399 -416). Eine MRT sollte insbesondere dann durchgeführt werden, wenn das Sprunggelenk röntgenologisch unauffällig ist und der Schmerz im Bereich des Innenknöchels länger als 1 Monat besteht (Menge T] et al. J Foot Ankle Surg 2015; 54: 242 - 246) [ Abb. 8]).

\section{Fibula}

Stressfrakturen der Fibula kommen je nach Literatur in ca. 1,3 bis 21,1 \% der Fälle vor, sodass hier ähnlich wie bei anderen Stressfrakturen von einer hohen Dunkelziffer auszugehen ist (Liong SY et al. Br J Radiol 2012; 85: 1148 -1156). Typischerweise ist das distale Drittel des Fibulaschafts betroffen, meist ca. $5-6 \mathrm{~cm}$ proximal des lateralen Malleolus. Deutlich seltener sind hingegen Stressfrakturen der proximalen Fibula (Harrast MA et al. Clin Sports Med 2010; 29: 399 - 416; Liong SY et al. Br J Ra-

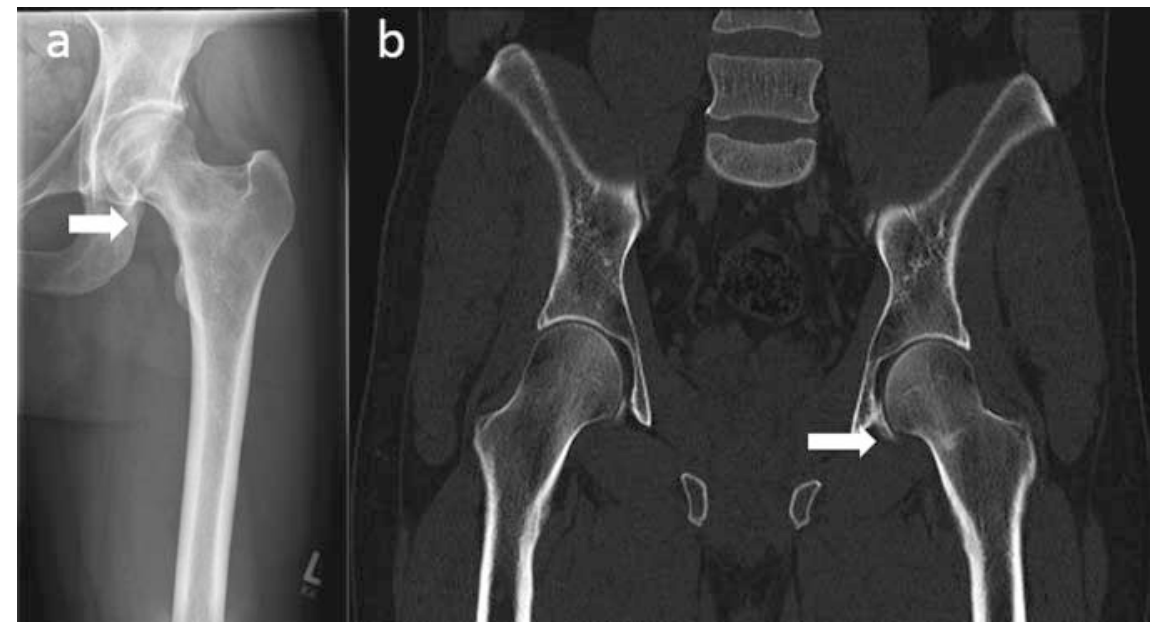

- Abb. 4 Konventionelles Röntgenbild a und koronare Reformation eines CT im Knochenfenster b. Es zeigt sich am medialen-inferioren Aspekt des Schenkelhalses eine Verdichtungslinie und Mehrsklerosierung (Pfeil), welche der Stressfraktur entspricht.
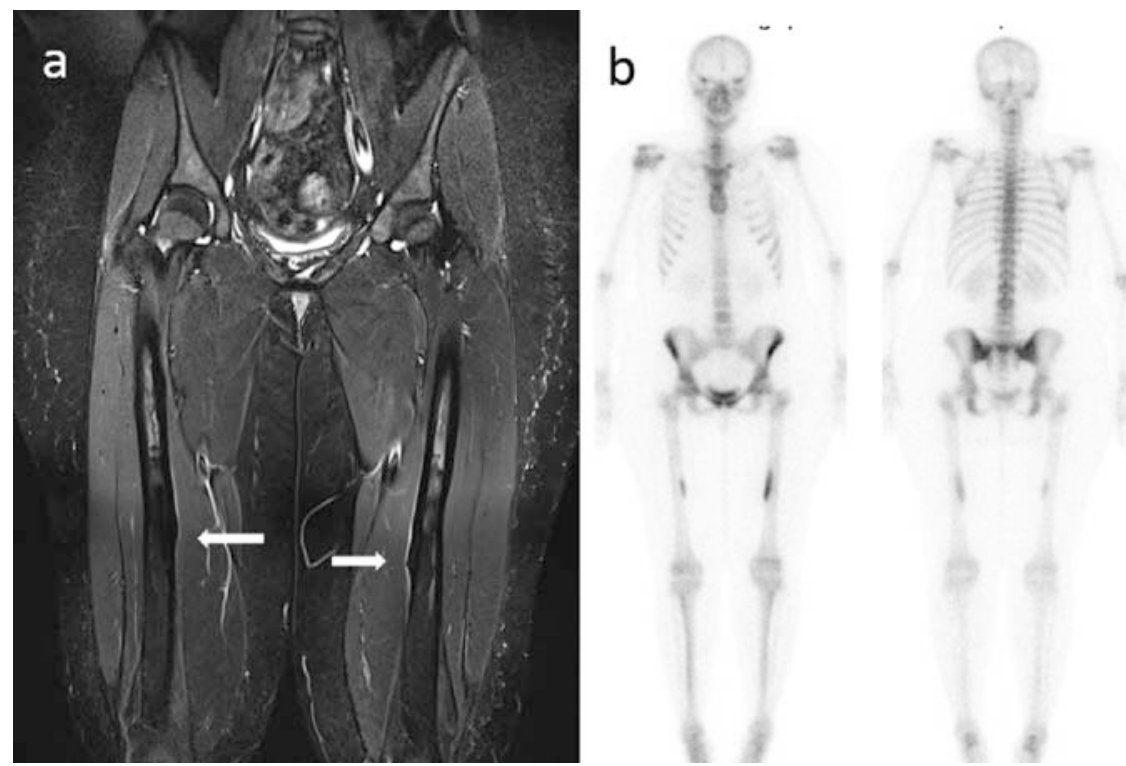

- Abb.5 MRT des Oberschenkels bds. a. Es zeigt sich in der koronaren fettunterdrückten PD ein fokales Knochenmarködem sowie eine kortikale Verdickung mit diskreter Periost-Reaktion im mittleren Drittel des Femurschafts (Pfeil), vereinbar mit einer beidseitigen Stressreaktion. Entsprechend zeigt sich in der Skelettszintigrafie eine fokale Mehranreicherung als Zeichen eines erhöhten Knochenstoffwechsels b.

diol 2012; 85: 1148 -1156). Klinisch geben die Patienten einen lokalisierten Druckschmerz im Bereich des lateralen distalen Unterschenkels an, meist ergeben sich anamnestisch Hinweise auf ein gesteigertes Laufpensum. Röntgenologisch initial meist okkult, kann im späteren Verlauf eine subtile Periost-Reaktion nachgewiesen werden. Die MRT hingegen lässt frühzeitig eine Sicherung der Diagnose zu.

\section{Fußwurzel}

Stressfrakturen der Tarsalia sind mit etwa 10 bis $25,3 \%$ an allen Stressfrakturen beteiligt (Liong SY et al. Br J Radiol 2012; 85: 1148 -1156). Der Calcaneus ist hier am häufigsten betroffen, meist in seinem posterioren-superioren Anteil (Liong SY et al. Br J Radiol 2012; 85: 1148 - 1156; Mandell JC et al. Skeletal Radiol 2017; 46: 1165 1186). Klinisch manifestiert sich die Stressfraktur des Calcaneus als Fersen- 


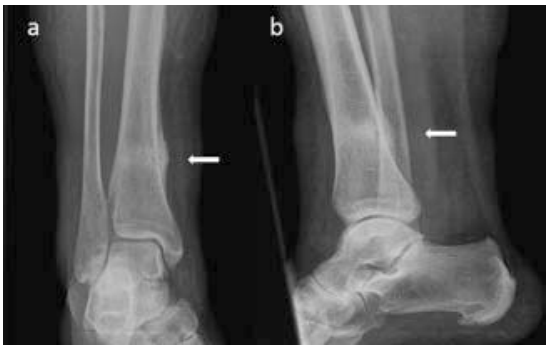

- Abb. 6 Konventionelle Röntgenbilder im ap-Strahlengang a und in lateraler Projektion b. Es zeigt sich eine partiell knöchern konsolidierte, horizontal verlaufende Stressfraktur in der distalen Tibia mit deutlicher Periost-Reaktion.

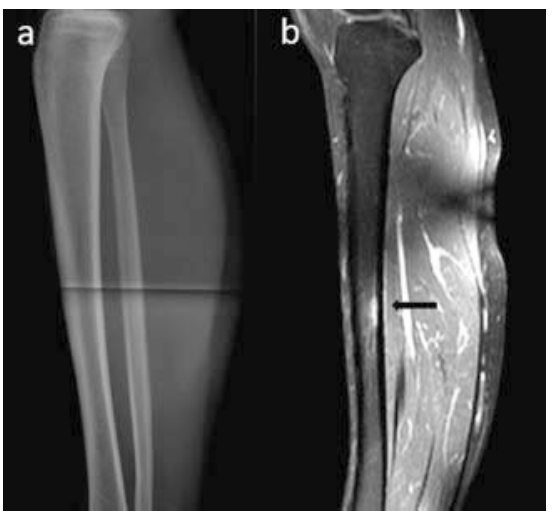

- Abb. 7 Konventionelles Röntgenbild im lateralen Strahlengang a und MRT desselben Unterschenkels in sagittaler Schnittführung mit fettunterdrückter PD-Wichtung $\mathbf{b}$. In der MRT zeigt sich ein fokales subkortikales Knochenmarködem mit begleitender Periost-Reaktion; im konventionellen Röntgenbild ist in diesem Stadium noch keine Auffälligkeit zu erkennen.

schmerz mit Druckschmerzhaftigkeit, welche beim Rennen oder Springen verstärkt wird (Liong SY et al. Br J Radiol 2012; 85: 1148 - 1156) [ $\bullet$ Abb. 9]). Röntgenologisch zeigt sich in der lateralen Projektion meist eine Verdichtungslinie senkrecht zur Verlaufsrichtung des trabekulären Knochens und parallel zur posterioren Kortikalis der Ferse.

Das Os naviculare ist mit ca. 2,4\% aller Stressfrakturen selten betroffen (Liong SY et al. Br J Radiol 2012; 85: $1148-1156$ ). Durch die Lage zwischen anteriorem Talus und den Ossa cuneiformia resultiert ein Impingement bei Stoßbelastungen entlang der Fußlängsachse und so ist wohl die Beteiligung insbesondere des zen-

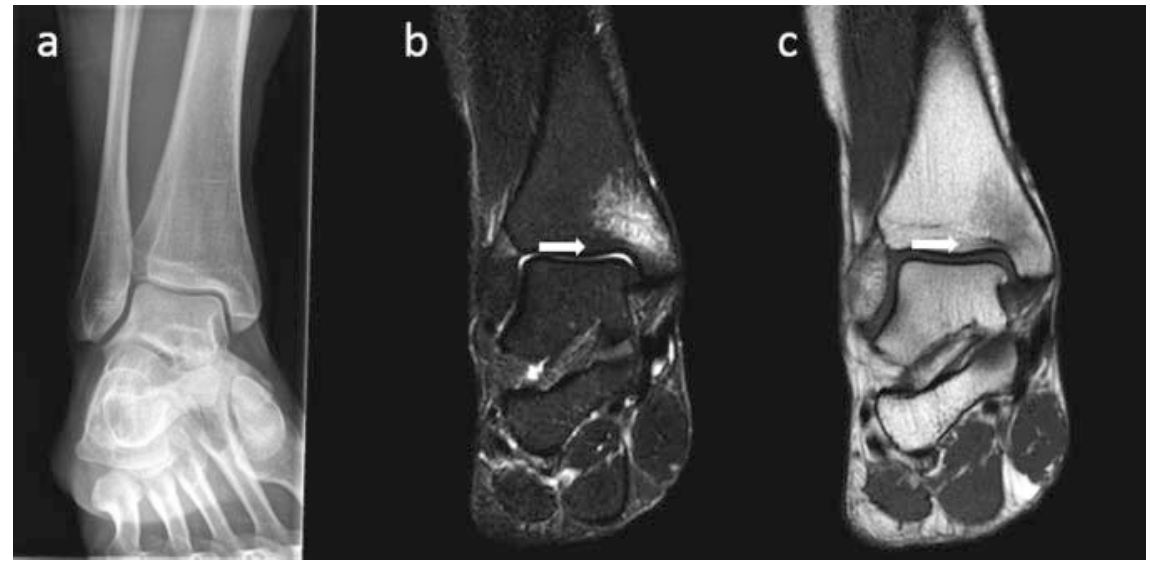

- Abb. 8 Stressfraktur des medialen Malleolus im konventionellen Röntgenbild a sowie im MRT mit Fettsättigung b und in der T1-Wichtung $\mathbf{c}$. Es zeigt sich ein Knochenmarködem an der Basis des medialen Malleolus mit feiner Frakturlinie (Pfeil) als Ausdruck der Stressfraktur.

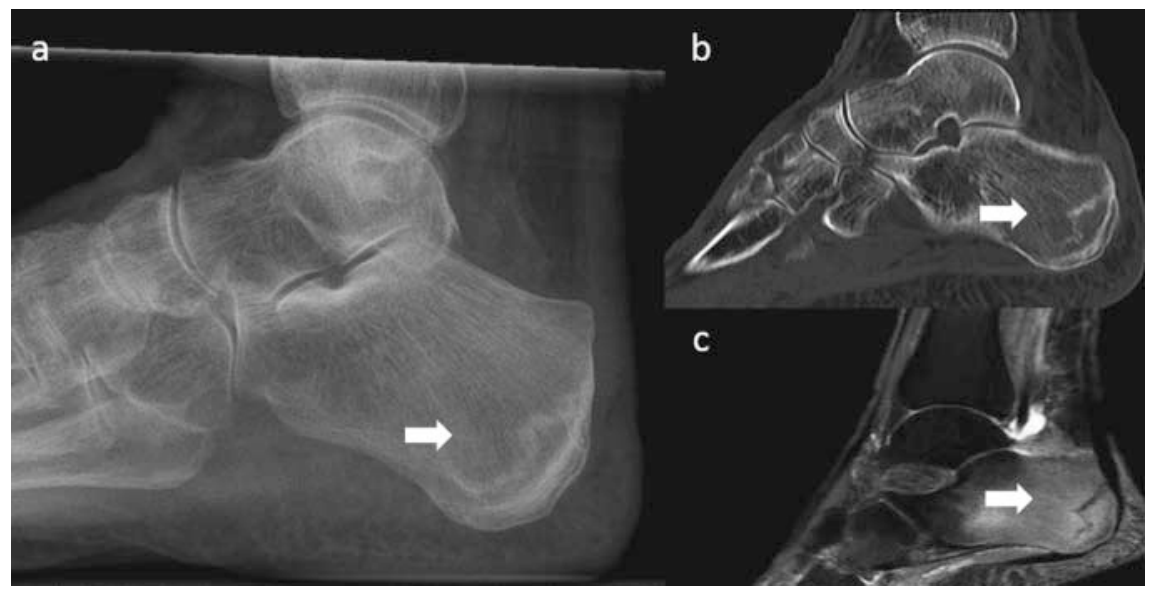

- Abb.9 Stressfraktur des Calcaneus im konventionellen Röntgenbild a, sagittaler Reformation im CT b und in der flüssigkeitssensitiven, fettgesättigten MRT-Sequenz c. Es zeigt sich eine trabekuläre Verdichtungslinie im posterioren Calcaneus (Pfeil), welche auch im CT und MRT erkennbar ist, mit begleitendem Knochenmarködem. tralen Anteils zu erklären (Harrast MA et al. Clin Sports Med 2010; 29: 399-416 [ $\vee$ Abb. 10]). Die klinische Diagnose bei unspezifischen Schmerzen im Bereich des Mittelfußes ist meist schwierig, die konventionelle Röntgendiagnostik mit einer Rate von $66 \%$ falsch negativen Befunden nicht zielführend, sodass weiterführende Schnitt-Bildgebung frühzeitig in Betracht gezogen werden muss (Liong SY et al. Br J Radiol 2012; 85: 1148 - 1156; Welck MJ et al. Injury 2017; 48: 1722 - 1726).

Der Talus ist im Rahmen von Stressfrakturen selten betroffen; wenn, dann finden sich Stressreaktionen meist im Bereich des Talus-Halses, Talus-Körpers und am lateralen Fortsatz (Liong SY et al. Br J Radiol 2012; 85: 1148 - 1156; Welck MJ et al. In-

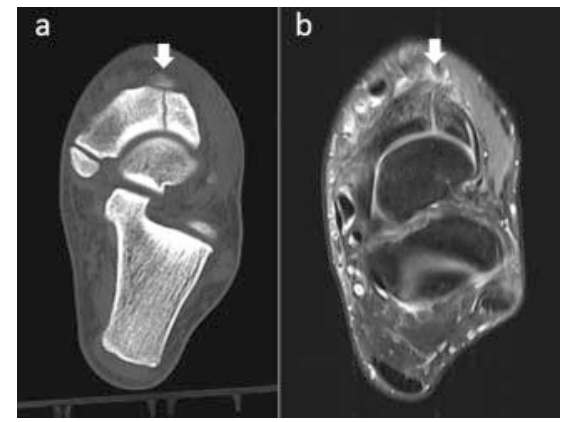

- Abb. 10 Nicht konsolidierte, vollständige Stressfraktur des Os naviculare, sichtbar als randlich mehrsklerosierte Frakturlinie in $\operatorname{der} \mathrm{CT} \mathbf{a}$ und MRT $\mathbf{b}$ mit angrenzendem Knochenmarködem. 


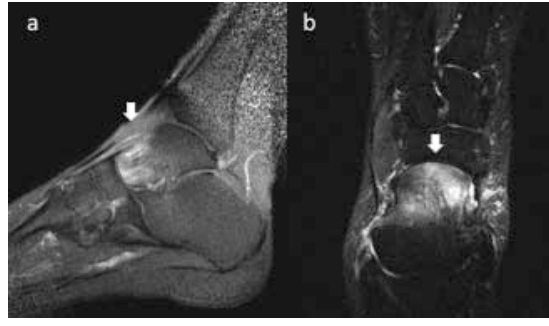

- Abb. 11 Die flüssigkeitssensitive MRT mit Fettunterdrückung zeigt in der sagittalen $\mathbf{a}$ und axialen Schichtführung $\mathbf{b}$ ein deutliches Knochenmarködem im anterioren Talus (Pfeil) ohne Nachweis höhergradiger arthrotisch-degenerativer Veränderungen; dies ist entsprechend als Stressreaktion bzw. inkomplette Stressfraktur zu werten.

jury 2017; 48: 1722 - 1726). Konventionell röntgenologisch sind die Stressreaktionen meist okkult, sodass die Diagnose nahezu ausschließlich mit MRT gestellt werden kann. Hier zeigt sich ein lokalisiertes Knochenmarködem und ggf. eine abgrenzbare Frakturlinie (Mandell JC et al. Skeletal Radiol 2017; 46: 1165 -1186) [ Abb. 11]).

\section{Metatarsalia}

Die Stressfraktur der Metatarsalia wird häufig als Marschfraktur bezeichnet, da sie initial bei Rekruten und später auch bei Läufern diagnostiziert wurde (Liong SY et al. Br J Radiol 2012; 85: 1148 - 1156). Sie stellt etwa 9 bis 24,6\% aller Stressfrakturen dar und kommt meist im mittleren bis distalen Drittel des Metatarsalschafts von Dig. 2 und 3 vor. Konventionell radiologisch zeigt sich eine periostale Reaktion oder auch eine diskrete Frakturlinie etwa 2 - 3 Wochen nach Symptombeginn; die MRT ist hier deutlich sensitiver und kann ein periostales Ödem, Knochenmarködem, angrenzende Weichteilveränderungen oder auch die Frakturlinie deutlich früher detektieren ( $\triangleright$ Abb. 12). Die Stressfraktur des Os metatarsale 5 ist mit $2 \%$ aller Stressfrakturen der Metatarsalia selten und liegt meist etwa $1,5 \mathrm{~cm}$ distal des Tuberkels. Sie muss klar von der AvulsionsFraktur des M. peroneus brevis abgegrenzt werden, welche auf Höhe des Tuberkels zu finden ist (Mandell JC et al. Skeletal Radiol 2017; 46: 1165 - 1186).

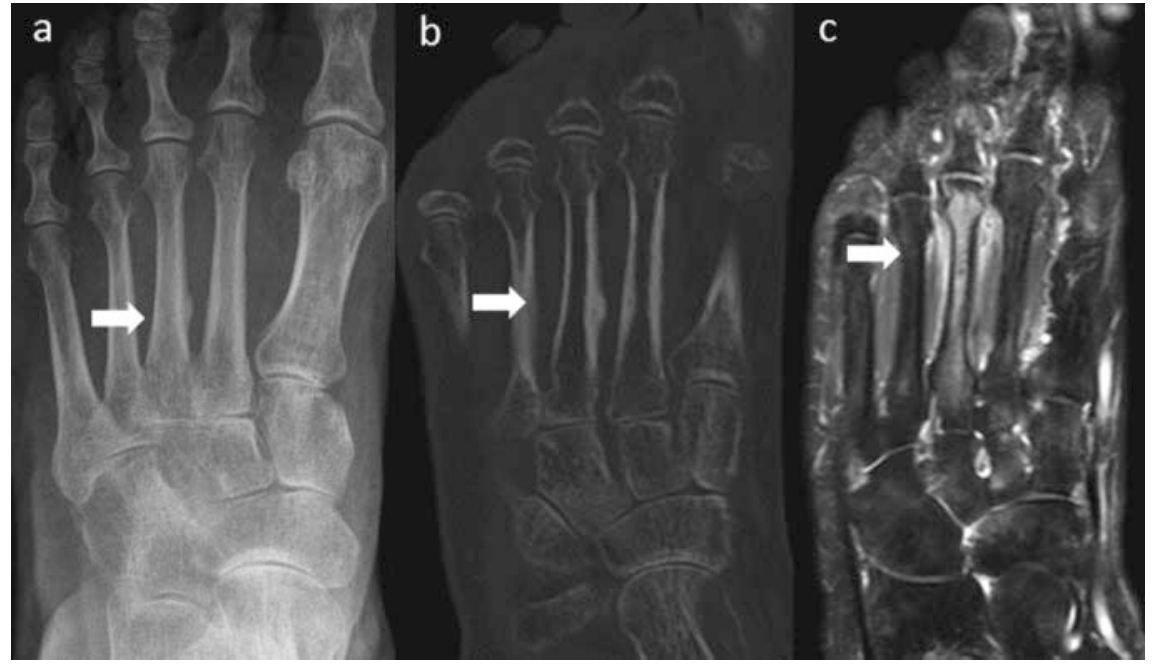

- Abb.12 Am Os metatarsale 3 zeigt sich sowohl im Röntgenbild a als auch in der CT b eine deutliche, fokale Periost-Reaktion mit kortikaler Verdickung bei hier bestehender Stressfraktur. Die MRT zeigt in den flüssigkeitssensitiven Sequenzen ein deutliches Knochenmarködem sowie eine ödematöse Mitreaktion der angrenzenden Weichteile c.

\section{Phalangen}

Stressfrakturen der Phalangen wurden bislang nur selten beschrieben und betreffen meist die Grundphalanx der Großzehe, allerdings sind auch andere Lokalisationen möglich. Patienten klagen über eine Schwellung und einen Druckschmerz im Bereich des Metatarsophalangeal-Gelenks, wenn konventionell-radiologisch noch kein Korrelat nachweisbar ist. Die MRT zeigt dann ein Knochenmarködem und ggf. eine feine Frakturlinie, sodass sie essenziell für die frühzeitige Diagnosesicherung ist (Yamaguchi S et al. Asia Pac J Sports Med Arthrosc Rehabil Technol 2017; 10: 12-16) [॰ Abb. 13]).

\section{Sesambein}

Seltene Stressfrakturen (etwa 1-3\% aller Stressfrakturen bei Athleten) betreffen die Sesambeine des M. flexor hallucis longus (Harrast MA et al. Clin Sports Med 2010; 29: 399 -416). Ursächlich können sie den mechanischen Belastungen und repetitiven Druckbelastungen bzw. Zugbelastungen bei forcierter Extension im Großzehengrundgelenk nicht standhalten, sodass es zu Stressreaktionen kommt (Harrast MA et al. Clin Sports Med 2010; 29: 399 -416; Mandell JC et al. Skeletal Radiol 2017; 46: 1165 -1186). Klinisch zeigt sich meist ein lokalisierter Schmerz und ggf. eine Schwellung im Bereich des Sesambeins. Konventionell-radiologisch

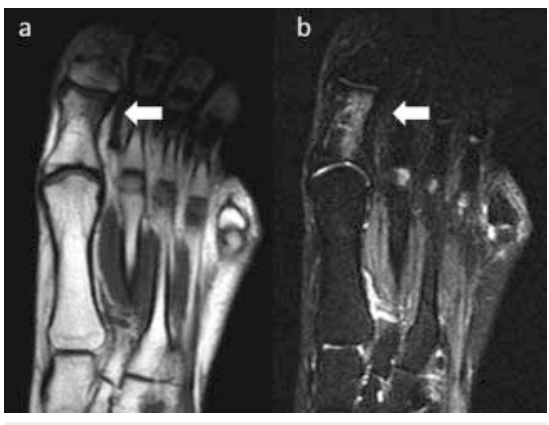

Abb.13 Die Grundphalanx der Großzehe zeigt in der MRT in T1-Wichtung a und in der flüssigkeitssensitiven Sequenz b ein Knochenmarködem im distalen lateralen Anteil, welches mit einer Stressreaktion bei vermehrter Belastung vereinbar ist. Eine wesentliche Mitreaktion der angrenzenden Weichteile besteht nicht.

kann sich eine quer verlaufende Aufhellung in der Schrägaufnahme zeigen; allerdings ist die Abgrenzbarkeit zum kongenital zweigeteilten Sesambein schwierig (Mandell JC et al. Skeletal Radiol 2017; 46: 1165 -1186). Hier hilft, wie so oft bei Stressreaktionen, die MRT weiter und zeigt ein entsprechendes Knochenmarködem bzw. ödematöse Veränderungen der Weichteile. 


\section{Zusammenfassung}

Überlastungsreaktionen und Stressfrakturen der unteren Extremität stellen eine wichtige klinische Entität dar und können sich in einer diffusen oder auch lokalisierten Beschwerdesymptomatik ohne eindeutig zuzuordnendes Trauma manifestieren. Aufgrund der in der Frühphase häufig fehlenden konventionell-radiologischen sichtbaren Veränderungen stellt hier die MRT die diagnostische Methode der Wahl dar. Die Kenntnis der typischen Lokalisationen an der unteren Extremität ist für die differentialdiagnostische Abgrenzung zu anderen Erkrankungen von entscheidender Bedeutung.
Interessenkonflikt

Die Autoren geben an, dass kein Interessenkonflikt besteht.

Autorinnen/Autoren

Fabian Springer, Marius Horger,

Bilal Al-Badayneh, Omar M. Albtoush

Department of Diagnostic and Interventional Radiology, University Hospital Tübingen,

Tübingen, Germany
Korrespondenzadresse

Dr. Omar M. Albtoush

Department of Diagnostic and Interventional Radiology, University Hospital Tübingen

Tübingen

Germany

o.albtoush@yahoo.com

Bibliografie

DOI https://doi.org/10.1055/a-0657-4137

Online-Publikation: 21.8.2018

Fortschr Röntgenstr 2019; 191: 173-178

(c) Georg Thieme Verlag KG, Stuttgart · New York ISSN 1438-9029 